\title{
Nutritional secondary hyperparathyroidism in two ponies
}

\author{
Luca Lacitignola ${ }^{1, *}$, Pasquale De Luca ${ }^{1}$, Rossella Santovito ${ }^{1}$, Maria Severa Di Comite ${ }^{2}$ and Antonio Crovace ${ }^{1}$ \\ ${ }^{l}$ Dipartimento dell'Emergenze e Trapianti di Organo,Sezione Cliniche Veterinarie e P.A., Università degli Studi di \\ Bari “Aldo Moro" s.p. per Casamassima Km 3, 70010, Valenzano, Bari, Italy \\ ${ }^{2}$ Dipartimento di Scienze mediche di base, neuroscienze e organi di senso, Sezione di Anatomia Umana ed Istologia, \\ Università degli Studi di Bari "Aldo Moro”, Piazza Giulio Cesare, Bari, Italy
}

\begin{abstract}
Nutritional secondary hyperparathyroidism (NSH) is uncommon in horses. Aim of this report is evaluate an alternative therapeutic approach and provide more information on the diagnostic procedures, through detailed microscopic findings of the bone lesions pony with NSH. Administration of tiludronate along with a balanced diet can be used in a treatment protocol for ponies with NSH. This case report suggests that tiludronate can be used to ameliorate the clinical signs of NSH.
\end{abstract}

Keywords: Hyperparathyroidism, Microradiography, Pony, Soft tissue mineralization, Tiludronate.

\section{Introduction}

NSH is identified as skeletal disease typical of animals fed a diet characterized by incorrect calciumphosphorus ratio food low in calcium and rich fodder oxalates, which bind calcium and not make it available for the absorption (Krook and Lowe, 1964; Joyce et al., 1971). In literature it is reported that Quarter Horse, Appaloosa and especially Pony have a greater incidence of this condition, in an age ranging from 2 to 8 years (Joyce et al., 1971).

Between 1905 and 1909 in South Africa have been reported 400 horses suffering from "big head disease", and in 1931 more than a quarter of the horses of the army hospital in the Philippines have also presented this condition, probably related to NSH nutritional source (Krook and Lowe, 1964). In a study conducted in the 1970s there was an increased incidence of NSH of origin food, with the presentation of 7 horses in 1 year affected by the pathology in question (Joyce et al., 1971).

The clinical symptoms of NSH and the resulting fibrous osteodystrophy in the horse are characterized by lameness, weakness, ataxia, and deformation of the bones of the skull (NSH, produce a condition called "big head disease". In more severe cases, the teeth can be loose, which together with increased volume of the maxilla and mandible, lead to chewing problems, anorexia, and cachexia (Frank et al., 1998).

The diagnosis of NSH is based on history, clinical signs, radiological findings, and laboratory testing. The main biochemical abnormalities consist of a decreased or normal concentration of ionized calcium in plasma and increased phosphate.
Increased ALP activity, probably caused by increased bone resorption, can also be detected. Urinalysis shows a low or normal calcium concentration and a marked hyperphosphaturia (Frank et al., 1998). The definitive diagnosis is obtained by measuring the serum parathyroid hormone level using the human radioimmunoassay that has been validated for the horse (Benders et al., 2001).

The purpose of this case series was to report the history, clinical findings, diagnostic methods, outcome of a novel therapy, and histopathological findings of 2 ponies with nutritional secondary hyperparathyroidism (NSH).

\section{Case 1}

\section{Case Details}

A 3-year-old male pony was referred in 2003 for severe dyspnea and deformity of the skull bones. The owner reported that the pony had manifested dyspnea and bony deformities since he had obtained the animal, about 1 month previously.

Upon clinical examination, the pony was found to exhibit generalized fatigue, and had severe dyspnea and inspiratory breath sounds. The temperature was $38.4^{\circ} \mathrm{C}$, heart rate $60 \mathrm{bpm}$, respiratory rate 36 breaths $/ \mathrm{min}$; the mucous membranes and capillary refill time were normal. The skull and jaw bones were extremely deformed, characterized by an abnormal imbalance between the transverse and longitudinal dimensions. Severe prognathism was apparent, and hardness of the palatine bones was found to be greatly diminished on palpation (Fig. 1A).

Radiographic examination revealed extreme thinning of the cranial bones with decreased density of 
medullary and cortical bone. Deviation of the nasal septum was also seen.

The left scapula, humerus, and proximal radius and ulna showed greatly decreased density, especially of cancellous bone; these findings were consistent with fibrous osteodystrophy.

The bicipital tendon insertions on the supraglenoid tubercle of the left scapula showed mineralized spurs (enthesophytosis) (Fig. 1B, C).

A standard biochemical panel showed hyperphosphatemia, normocalcemia, and a high alkaline phosphatase (ALP) level. A radioimmunoassay showed an increased parathyroid hormone (PTH) level (Table 1). The clinical, radiographic, and biochemical findings indicated that the pony had fibrous osteodystrophy secondary to $\mathrm{NSH}$. The dietary cause was based on the medical history and findings of normocalcemia and hyperphosphatemia.

The pony received tiludronate (Tildren $®)(1 \mathrm{mg} / \mathrm{kg})$ administered intravenously over a 30 -min period as a single dose diluted in $500 \mathrm{ml}$ of $0.9 \%$ saline.

Follow-up laboratory examinations and radiography were performed 30 days after the administration of tiludronate. There were no noticeable changes in biochemical parameters except for a significant decrease in the ALP level. PTH was re-evaluated and was unchanged. Radiological findings were not appreciably different from before administration of tiludronate.

By contrast, the pony's clinical condition was markedly improved, with resolution of the clinical signs (asthenia, anorexia, inability to remain standing, lameness, and respiratory sounds). The pony was discharged.

Table 1. Laboratory values of case 1 at the time of presentation and 30 days after tiludronate therapy.

\begin{tabular}{cccc}
\hline Parameter & $\begin{array}{c}\text { Normal } \\
\text { Values }\end{array}$ & Presentation & Day 30 \\
\hline $\begin{array}{c}\text { Alkaline } \\
\text { Phosphatas } \\
\text { (IU/L) }\end{array}$ & $86-285$ & 634 & 483 \\
$\begin{array}{c}\text { BUN } \\
(\mathrm{mg} / \mathrm{dL})\end{array}$ & $20-35$ & 16 & $\mathrm{~N} / \mathrm{A}$ \\
$\begin{array}{c}\mathrm{Ca} \\
(\mathrm{mg} / \mathrm{dL})\end{array}$ & $10.1-13.7$ & 10.41 & 10.91 \\
$\begin{array}{c}\mathrm{Creatinine} \\
(\mathrm{mg} / \mathrm{dL})\end{array}$ & $0.8-1.8$ & 0.98 & N/A \\
\hline $\begin{array}{c}\text { Ionized Ca } \\
(\mathrm{mM})\end{array}$ & $1.4-1.7$ & 1.7 & 1.68 \\
$\begin{array}{c}\mathrm{P} \\
(\mathrm{mg} / \mathrm{dL})\end{array}$ & $1.5-4.5$ & 5.1 & 4.4 \\
$\begin{array}{c}\text { PTHi } \\
(\mathrm{pg} / \mathrm{mL})\end{array}$ & $75-95$ & 567 & 510 \\
\hline $\mathrm{N} / \mathrm{A}$ Data & & &
\end{tabular}

N/A: Data not available.

\section{Case 2}

A 5-year-old male Shetland pony was referred in October 2012, with a history of progressive asthenia and 3 days of recumbency. The morning he was seen, he had been found recumbent, with nystagmus, profuse sweating, and tenderness of the left hind limb. The owner reported that he had administered flunixin meglumine and corticosteroids to the pony, and that the animal was exclusively fed a wheat bran diet. A general clinical examination showed persistent recumbency, slight enlargement of the facial bones, pain upon flexion of the hind limbs, and pale mucous membranes. The temperature was $37.7^{\circ} \mathrm{C}$, heart rate $44 \mathrm{bpm}$, and respiratory rate 32 breaths/min. Neurological examination was normal except from nystagmus.

Radiography was performed at the level of the stifle joint and tibia. There was diffuse bone resorption of the distal femur, proximal tibia, proximal portion of the patella, tibial diaphysis, and femoral trochlea. Osteophytes were seen at the level of the lateral femoral condyle and enthesophytes at the insertion of the medial and lateral collateral ligaments of the stifle joint and at the distal tibia. The radiological findings were compatible with fibrous osteodystrophy and soft tissue mineralization (Fig. 1D, E, F).

Laboratory testing showed a marked increase in the ALP level, and slight hypocalcemia associated with normophosphatemia. The PTH level was increased over the instrument detectable range. The clinical, radiographic, and biochemical findings indicated that the pony had fibrous osteodystrophy secondary to NSH. A dietary cause was based on the nutritional history and finding of hypocalcemia (Table 2).

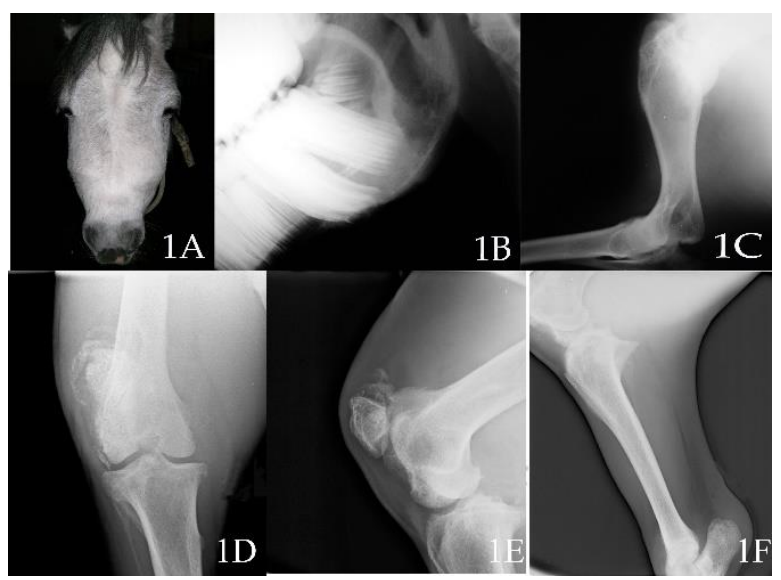

Fig. 1. (A, B and C): Clinical aspect and X-rays of case 1 . (A): show severe enlargement of cranial bones. (B): Lateral view of mandible, note radiotrasparency of cancellous bone. (C): Medio-Lateral view of proximal front limb, with bone loss consistency and soft tissue mineralization. (D, E and F): $\mathrm{X}$-rays of Case 2. (D and E): respectively $\mathrm{CdCr}$ view and LM oblique view of stifle joint. (F): LM view of tibia. Notable soft tissue mineralization and bone demineralization. 
Table 2. Laboratory values of case 2 at the time of presentation. No additional evaluations were performed, because the pony was euthanized.

\begin{tabular}{ccc}
\hline Parameter & Normal Values & Presentation \\
\hline $\begin{array}{c}\text { Alkaline Phosphatase } \\
(\mathrm{IU} / \mathrm{L})\end{array}$ & $86-285$ & 1499 \\
$\begin{array}{c}\mathrm{BUN} \\
(\mathrm{mg} / \mathrm{dL})\end{array}$ & $20-35$ & 48 \\
$\begin{array}{c}\mathrm{Ca} \\
(\mathrm{mg} / \mathrm{dL})\end{array}$ & $10.1-13.7$ & 10.2 \\
$\begin{array}{c}\text { Creatinine } \\
(\mathrm{mg} / \mathrm{dL})\end{array}$ & $0.8-1.8$ & 1.4 \\
\hline $\begin{array}{c}\text { Ionized Ca } \\
(\mathrm{mM})\end{array}$ & $1.4-1.7$ & $\mathrm{~N} / \mathrm{A}$ \\
$\begin{array}{c}\mathrm{P} \\
(\mathrm{mg} / \mathrm{dL})\end{array}$ & $1.5-4.5$ & 4.3 \\
$\begin{array}{c}\mathrm{PTHi} \\
(\mathrm{pg} / \mathrm{mL})\end{array}$ & $75-95$ & $>2000$ \\
\hline
\end{tabular}

N/A: Data not available.

The pony was administered intravenous fluid therapy $(10 \mathrm{ml} / \mathrm{kg} / \mathrm{h})$ to compensate dehydration. To prevent excessive calcium loss do to high sodium containing fluid, calcium gluconate $20 \%$ was added in a total dose of $250 \mathrm{ml}$ in two hours; flunixin meglumine $(1 \mathrm{mg} / \mathrm{kg})$, and phenylbutazone $(4.4 \mathrm{mg} / \mathrm{kg})$.

On the third day of treatment, the pony's clinical condition became hopeless due to a pathological fracture of right humerus occurred going to standing position. No further treatment was performed, and the pony was euthanized according with the owner. Bone samples were harvested for further study.

The humeral epiphyses were fixed using $4 \%$ paraformaldehyde in $0.01 \mathrm{M}$ phosphate-buffered saline (PBS, pH 7.2) for 2 days at $4^{\circ} \mathrm{C}$, washed in running water for 2 hours, dehydrated in ethanol, and conventionally processed for methylmethacrylate embedding. Fifteen- $\mu \mathrm{m}$ serial sections were cut in the frontal plane using a circular diamond-coated Leica SP1600 saw microtome (Leica Biosystems), polished under running water, and air dried for 24 hours in an incubator at $37^{\circ} \mathrm{C}$. The sections were then microradiographed at $8 \mathrm{kV}$ and $14 \mathrm{~mA}$ using an XRG3000 x-ray generator (Ital Structures Research; Riva Del Garda, Italy). The contact high-resolution microradiographs were developed using Kodak HC110 Developer, fixed in Ilford Hypam Rapid Fixer, washed in double-distilled water, and air dried at room temperature. Nondecalcified sections were stained using $1 \%$ toluidine blue $(\mathrm{pH}$ 3.7). Histological evaluations were performed using a Nikon Eclipse E400 light microscope coupled with a DS-5M digital camera (Nikon Corporation, Tokyo, Japan).
Microradiographs of the frontal serial sections showed modifications in the arrangement of the bone tissue and mineralization. The trabecular network appeared rarefied because of thinning or complete erosion of the trabeculae.

The subchondral compact bone also contained large erosion cavities, and reduced density was apparent. Both spongy and compact bone frequently exhibited large osteocytic lacunae. In addition, new less mineralized bone was extensively present. The new matrix was laid down upon the surface of pre-existing bone or appeared as new small, fragmented trabeculae (Fig. 2).

The stained sections of bone showed numerous active osteoclasts on the erosion surfaces, and wide osteocytic lacunae with osteocytes surrounded by a metachromatic matrix were seen in the adjacent mineralized matrix. Mast cells were found in the bone marrow and near the osteoclasts, where the mast cells released metachromatic granules.

The mineralization fronts showed wide osteoid seams containing many osteoblasts, or occasionally numerous preosteoblasts with fibroblastic morphology. Fibrous connective tissue frequently covered the trabecular surfaces and replaced some of the reticular stroma of the marrow.

Finally, a patchwork of numerous irregular bone fragments, stained and oriented differently, caused some trabeculae to take on a characteristic mosaic appearance (Fig. 3).

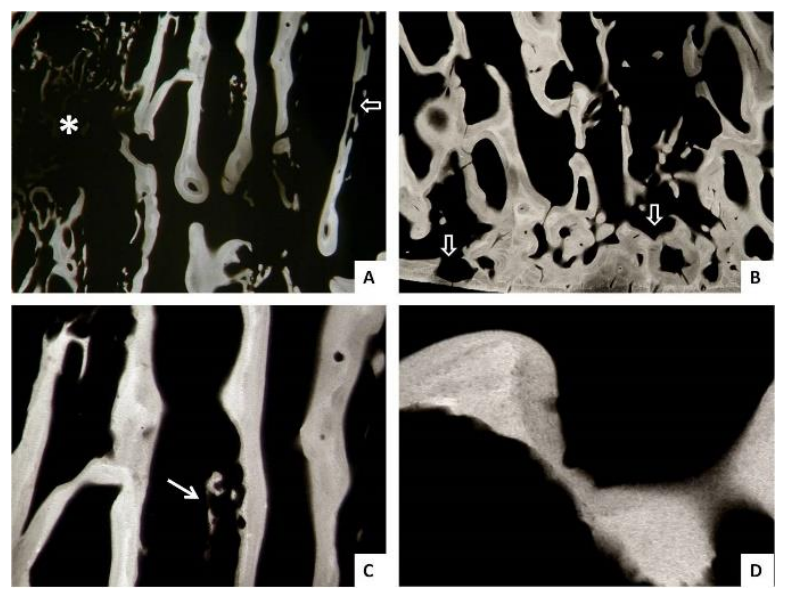

Fig. 2. Contact microradiographs: poorly mineralized matrix appears more radiotransparent than normal matrix. (A and B): bone architecture is modified because of increased trabecular loss (asterisk) and large areas of surface erosion (empty arrows) affecting spongy and subchondral bone. (C): thin trabeculae (thin arrow) are forming in front of Howship's lacunae. (D): the erosion and, on the opposite side of the same trabecula, a deposition front, reflect high bone turnover. Large osteocytic lacunae are visible. [magnification: (A and B): 4x; (C): 10x; (D): 20x]. 

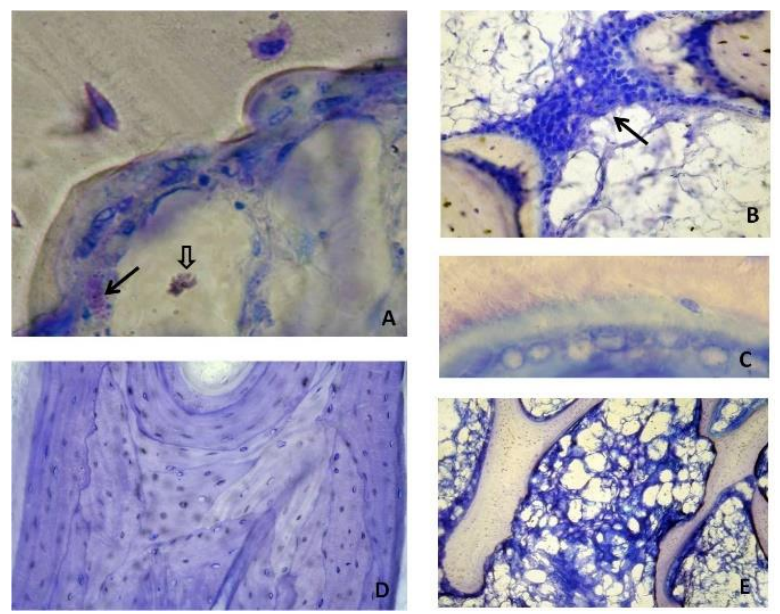

Fig. 3. Nondemineralized bone sections (toluidine blue stain). (A): osteoclasts and mast cell showing extrusion of metachromatic granules (thin arrow). A round mast cell in the bone marrow (empty arrow). (B): plump fibroblast-like preosteoblasts (arrow) surround a poorly organized bone matrix. (C): bone-forming surface with a wide unmineralized osteoid seam lined by osteoblasts. (D): numerous cement lines delimit the bone fragments, giving a mosaic appearance to the section. (E): the marrow spaces are replaced by fibrocellular tissue. [magnification: (A and C): 40x; (B and E): 10x; (D): 20x].

\section{Discussion}

The clinical manifestations observed in the course of this condition are a result of fibrous osteodystrophy due to sustained release of PTH. Because of increased bone resorption, the structural components of the bone are reduced, and with the subsequent attachment of fibrous tissue, result in weakness and deformities of the involved bones (Coffman, 1980).

Our first clinical case manifested the characteristic signs of NSH, presenting with lameness and reluctance to move, and obvious deformities of the bones of the skull. It was also interesting to note the strong respiratory sounds and the radiographic abnormalities and deformities of the facial bones.

The bone lesion associated with $\mathrm{NSH}$, fibrous osteodystrophy, is easily diagnosed by radiography, and the diagnosis can be confirmed by postmortem histological examination of the involved bones. Radiographic findings include generalized loss of bone density and reduced definition of bone trabeculae, which have a coarse appearance. In addition sometimes shoulder and ribs have an osteoporotic appearance (Benders et al., 2001). Radiological examination of both cases found diffuse bone resorption and enthesophytes . This important diagnostic finding was due to mineralization of the soft tissues and entheses. These findings were described as sites of mineralization in human beings and animals, even in the horse, affected by primary or secondary hyperparathyroidism (Hatt and Sainsbury, 1998; Estepa et al., 2006; Staszków et al., 2013).

Radiographic examination of the first case revealed marked alterations at the level of the shoulder and elbow joints, while the second case had major alterations at the level of the femorotibial and femoropatellar joint and the patella. Therefore, in these types of cases, there are strong indications for performing radiographic examination of the epiphyses, which are the primary location of cancellous bone, the first type of bone affected by resorption.

To make a definitive diagnosis of NSH, a biochemical profile and PTH level are needed (Jubb and Kennedy, 1970; Estepa et al., 1998). The results of the biochemical profile of our first case were indicative of the pathology in question; the pony presented with a very high ALP level, normocalcaemia, and hyperphosphatemia. The PTH level was markedly increased compared to the reference range. An increased ALP level is associated with increased bone resorption and in according to the characteristics of normocalcemia and hyperphosphatemia of NSH. It has been thought that normocalcaemia and hyperphosphatemia reflect the nutritional origin of the pathology in question, because both primary hyperparathyroidism and the secondary hyperparathyroidism of renal origin have been reported to show an increase in plasma calcium. With secondary hyperparathyroidism of renal origin, there is also an increase in the plasma creatinine and urea levels because of kidney damage (Joyce et al., 1971; Coffman, 1980; Arnaud, 1994).

The treatment of horses with NSH includes correcting the calcium and phosphorus ratio in the diet using calcium and phosphorus supplements and treating the skeletal manifestations. Horses with severe skeletal deformities, including the ponies we have described, are unable stand, or at least do so with difficulty because of the severe bone pain. Nonsteroidal antiinflammatories are useful in these cases, but in any case the prognosis is poor.

Tiludronate, which is a bisphosphonate, is approved for use in the equine species. Tiludronate has been reported to be effective in horses 1 month after starting treatment for common bone lesions characterized by osteolysis, such as navicular disease, osteoarthritis, enthesitis, and subchondral bone cysts (Denoix et al., 2003; Coudry et al., 2007; Gough et al., 2010). Indeed, there was noticeable clinical improvement in our first case, with the disappearance of lameness and regained ability to remain standing within 30 days after tiludronate infusion. There was also a marked decrease in the ALP level, although the PTH level did not change appreciably, and repeat radiography did not demonstrate any appreciable changes. 
Tiludronate was not administered to the second pony because of severe humeral fracture and because the euthanasia according with the owner.

To evaluate the bone lesions manifested in fibrous osteodystrophy, histological and microradiographic examinations were performed on postmortem bone samples after the pony was euthanized. Typically fibrous osteodystrophy microscopic findings include extensive resorption of the cortical and subchondral bone, which appear as Howship's lacunae containing multinucleated osteoclasts (Joyce et al., 1971). In our evaluation, the affected bone is replaced by abundant fibrocellular tissue and a few newly formed bone trabeculae lined by osteoblasts, or dense fibrous tissue with fibroblasts arranged in spirals or columns. Periosteal new bone formation and deposition of nonmineralized osteoid covered by osteoblasts may also be seen.

The findings confirmed the diagnosis and provided in depth pathological information on this condition, which had been previously studied in humans and rarely in horses. In another case report have been reported similar findings in histomorphometric analysis only by HEE Staining. Our peculiarity in microscopic evaluation is to give more information by the use of microradiography and toluidine blue staining, not previously described with horses affected by fibrous osteodystrophy secondary to NSH.

To the best of our knowledge, this is the first report describing the microradiographic appearance of the bone lesions of fibrous osteodystrophy in the horse. In addition, this report presented the histological evidence that confirmed the clinical diagnosis of fibrous osteodystrophy. The coadministration of the Tiludronate with a balanced diet can be used for treating and ameliorate prognosis for horses with $\mathrm{NSH}$, although may not lead to resolution of the deformities. Conflict of interest

The authors declare that there is no conflict of interests.

\section{References}

Arnaud, C.D. 1994. The calciotropic Hormones and metabolic bone disease. In: Greenspan FS; Baxter JD, eds. Basic and clinical endocrinology. $4^{\text {th }}$ ed. Norwalk, Conn: Appleton Lange, pp: 227-306.

Benders, N.A., Junker, K., Wensing, Th., Van Den Ingh, Th. and Van Der Kolk, J.H. 2001. Diagnosis of NSH in a pony using intact parathyroid hormone radioimmunoassay. Vet. Rec. 149(6), 185-187.
Coffman, J. 1980. Calcium and phosphorus physiology and patho-physiology. Vet. Med. Small Anim. Clin. 75(1), 93-96.

Coudry, V., Thibaud, D., Riccio, B., Audigié, F., Didierlaurent, D. and Denoix, J.M. 2007. Efficacy of tiludronate in the treatment of horses with signs of pain associated with osteoarthritic lesions of the thoracolumbar vertebral column. Am. J. Vet. Res. 68(3), 329-337.

Denoix, J.M., Thibaud, D. and Riccio, B. 2003. Tiludronate as a new therapeutic agent in the treatment of navicular disease: a double-blind placebo-controlled clinical trial. Equine Vet. J. 35(4), 407-413.

Estepa, J.C., Aguilera-Tejero, E., Zafra, R., MayerValor, R., Rodríguez, M. and Perez, J. 2006. An Unusual Case of Generalized Soft-Tissue Mineralization in a Suckling Foal. Vet. Pathol. 43(1), 64-67.

Estepa, J.C., Aguilera-Tejero, E., Mayer-Valor, R., Almeden, Y., Felsenfeld, A.J. and Rodriguez, M. 1998. Measured of parathyroid hormone in horses. Equine Vet. J. 30, 476-481.

Frank, N., Hawkins, J.F., Couetil, L.L. and Raymond, J.T. 1998. Primary Hyperparathyroidism with osteodystrophia fibrosa of the facial bones in a pony. J. Am. Vet. Med. Assoc. 212, 84-86.

Gough, M.R., Thibaud, D. and Smith, R.K. 2010. Tiludronate infusion in the treatment of bone spavin: a double blind placebo-controlled trial. Equine Vet. J. 42(5), 381-387.

Hatt, J.M. and Sainsbury, A.W. 1998. Unusual case of metabolic bone disease in a common marmoset (Callithrix jacchus). Vet. Rec. 143(3), 78-80.

Joyce, J.R., Pierce, K.R., Romane, W.M. and Baker, J.M. 1971. Clinical study of nutritional NSH in horses. J. Am. Vet. Med. Assoc. 158, 2033-2042.

Jubb, K.V.F. and Kennedy, P.C. 1970. Pathology of Domestic Animals. Vol. 1, $2^{\text {nd }}$ ed. Academic Press, New York, pp: 35-37.

Krook, L. and Lowe, J.E. 1964. Nutritional Secondary Hyperparathyroidism in the Horse: With a Description of the Normal Equine Parathyroid Gland. Pathol. Vet. suppl 1, 1-98.

Staszków, M., Wojtaszek, E., Żebrowski, P. and Matuszkiewicz-Rowińska, J. 2013. Massive soft tissue calcifications in severe hyperparathyroidism secondary to end-stage renal disease. Pol. Arch. Med. Wewn. 123(4), 191-192. 